\title{
Serum Eicosapentaenoic Acid/Arachidonic Acid Ratio Is Low in Patients with Pulmonary Thromboembolism
}

\author{
Yuichi Oshima ${ }^{1}$, Katsuyuki NiкI ${ }^{1}$, Yoshimune HIRAмото ${ }^{1}$, Shinji MоRIмото ${ }^{1}$, \\ Hiroshi TAKAMI $^{2}$ and Masahiro IZUMI ${ }^{1}$ \\ ${ }^{1}$ Division of Cardiology, Department of Internal Medicine, and ${ }^{2}$ Department of General Medicine, \\ Kinki Central Hospital, 3-1 Kuruma-zuka, Itami, Hyogo 664-8533, Japan \\ (Received March 21, 2013)
}

\begin{abstract}
Summary Recently $n-3$ polyunsaturated fatty acids (PUFAs) have been reported to play protective roles against cardiovascular diseases. Pulmonary thromboembolism (PTE) is one of the critical diseases in the circulatory system. However the relationship between $n-3$ PUFAs and PTE has not been reported. A total of 144 outpatients of the division of cardiology, including 12 of PTE cases, were enrolled in the present study and serum levels of eicosapentaenoic acid (EPA) and arachidonic acid (AA) were analyzed. We found that the EPA/AA ratio of the patients with PTE (the PTE group) was significantly lower than that of the patients without PTE (the non-PTE group) ( $p=0.007$ for $\log \mathrm{EPA} / \log \mathrm{AA}$ ratio). Next, the PTE group was divided into two groups by the presence or absence of malignant carcinoma. The PTE without carcinoma group showed significantly lower EPA/AA ratio than that of the non-PTE group ( $p=0.002$ for $\log$ EPA/log AA ratio). However, the PTE with carcinoma group did not show the statistical difference in EPA/AA ratio compared with the non-PTE group ( $p=0.39$ ). These data indicate that PTE may be associated with a low EPA/AA ratio, although the presence of malignant carcinoma should be taken into account.
\end{abstract}

Key Words polyunsaturated fatty acids, eicosapentaenoic acid, pulmonary thromboembolism, EPA/AA ratio

Accumulating studies have shown that $n-3$ polyunsaturated fatty acids (PUFAs) have protective properties against cardiovascular diseases (CVD) (1-3). A study has indicated that high intake of $n-3$ PUFAs is one possible reason for the relatively low prevalence of CVD in Japan (4); however, it was reported that the relative ratio of n-3 PUFA intake in Japan was decreasing (5).

Pulmonary thromboembolism (PTE) is one of the critical circulatory diseases, in which the thrombi usually originate in the deep veins of the lower limbs. The incidence of PTE in Japan was relatively low in the past, but it is increasing as a westernized lifestyle replaces the traditional one.

These data may suggest a link of $n$-3 PUFAs with PTE. However there have been no clinical studies concerning the relationship between $n-3$ PUFAs and PTE, which led us to study serum fatty acid composition in relation to PTE prevalence.

\section{Materials and Methods}

From July 2011 to October 2011, among the outpatients who visited the Division of Cardiology in our hospital, a total of 144 patients who received blood sampling tests for serum fatty acid composition were enrolled in the present study. The present study was approved by the ethical committee in Kinki Central Hos-

E-mail: ooshima_y@kich.itami.hyogo.jp pital and carried out in accordance with the code of ethics of the World Medical Association (Declaration of Helsinki).

All patients underwent blood sampling after more than an 8-h fasting period in the morning. Serum fatty acid composition was determined by the gas-chromatography method performed by SRL Inc, Tokyo, Japan. For the statistical analyses, the data for the fatty acid composition were log-transformed to be in the normal distribution.

The diagnosis of PTE was done by the detection of pulmonary arterial thrombi by chest contrast enhanced computed tomography (CE-CT). Outpatients who did not have any echocardiographic signs of pulmonary hypertension, clinical history of PTE or clinical signs for PTE did not undergo the chest CE-CT examination because they were unlikely to have PTE. The PTE patients were reported negative for coagulation disorders, including anti-phospholipid syndrome, protein $\mathrm{C}$ deficiency, protein $\mathrm{S}$ deficiency and antithrombin 3 deficiency.

Data are shown as means \pm SE. Comparisons between two groups were done with Student's t-test for the continuous variables, and the Chi-square test with Yates' correction or Fisher's exact test for the categorical data. For multiple comparisons, one-way analysis of variance (ANOVA) with Bonferroni correction was performed by using the StatView computer program (Version 5.0). The $p$ values $<0.05$ were regarded as statistically significant. 
Table 1. General clinical characteristics of the cohort.

\begin{tabular}{lc}
\hline Male/female & $99 / 45$ \\
Age (y old) & $67.8 \pm 1.0$ \\
Body mass index $\left(\mathrm{kg} / \mathrm{m}^{2}\right)$ & $24.3 \pm 0.3$ \\
High blood pressure $(\%)$ & 79.9 \\
Dyslipidemia $(\%)$ & 77.8 \\
Diabetes mellitus (\%) & 43.8 \\
Chronic kidney disease (\%) & 43.1 \\
Cerebral vessel disease (\%) & 18.1 \\
Ischemic heart disease (\%) & 37.5 \\
Reduced LV function (\%) & 5.6 \\
Valvular disease (\%) & 14.6 \\
Arteriosclerosis obliterans (\%) & 8.3 \\
Atrial fibrilation (\%) & 10.4 \\
Pulmonary thromboembolism (\%) & 8.3 \\
\hline
\end{tabular}

LV: left ventricle.

Table 2. Comparisons of the clinical characteristics between non-PTE and PTE groups.

\begin{tabular}{lccc}
\hline & $\begin{array}{c}\text { non-PTE } \\
(n=132)\end{array}$ & $\begin{array}{c}\text { PTE } \\
(n=12)\end{array}$ & $p$ value \\
& $93 / 39$ & $6 / 6$ & 0.255 \\
Male/female & $67.9 \pm 1.0$ & $66.3 \pm 4.2$ & 0.723 \\
Age (y old) & $24.2 \pm 0.3$ & $24.9 \pm 2.1$ & 0.746 \\
BMI (kg/m $\left.{ }^{2}\right)$ & $132.6 \pm 1.7$ & $134.4 \pm 5.6$ & 0.766 \\
SBP (mmHg) & $75.8 \pm 1.1$ & $76.3 \pm 4.2$ & 0.915 \\
DBP (mmHg) & 84.1 & 33.3 & $<0.0001$ \\
HBP (\%) & 78.8 & 66.7 & 0.292 \\
Dyslipidemia (\%) & 44.7 & 33.3 & 0.549 \\
Diabetes mellitus (\%) & 43.2 & 41.7 & 1.000 \\
Chronic kidney disease (\%) & 19.7 & 0 & 0.124 \\
Cerebral vessel disease (\%) & 39.4 & 16.7 & 0.132 \\
Ischemic heart disease (\%) & 3.1 & 0 & 1.000 \\
Reduced LV function (\%) & 6.1 & 16.7 & 1.000 \\
Valvular disease (\%) & 14.4 & 0 & 0.577 \\
ASO (\%) & 9.1 & 0 & 0.443 \\
Atrial fibrilation (\%) & 11.4 & 50 & $<0.0001$ \\
carcinoma (\%) & 4.5 & & \\
& & &
\end{tabular}

BMI: body mass index, LV: left ventricle, SBP: systolic blood pressure, DBP: diastolic blood pressure, ASO: arteriosclerosis obliterans.

\section{Results and Discussion}

All participants' characteristics are shown in Table 1. Male patients were twice as many as female ones. Twelve $(8.3 \%)$ patients had PTE in this cohort. We compared the background characteristics between the patients without PTE (non-PTE) and those with PTE (Table 2). There are no differences in age, body mass index or blood pressure. In the PTE group, half of the patients had carcinoma $(p<0.0001$ as compared with non-PTE group).

As shown in the biochemical analyses (Table 3), the EPA value and EPA/AA ratio of the PTE group were lower than those of non-PTE group $(p=0.017$ for $\log$ EPA and 0.007 for $\log$ EPA/log AA ratio).

As for the age and the EPA/AA ratio, the present cohort is comparable to that of the recent report on
Table 3. Comparisons of the biochemical parameters between non-PTE and PTE groups.

\begin{tabular}{lccc}
\hline & $\begin{array}{c}\text { non-PTE } \\
(n=132)\end{array}$ & $\begin{array}{c}\text { PTE } \\
(n=12)\end{array}$ & $p$ value \\
& $101.9 \pm 2.7$ & $104.6 \pm 14.7$ & 0.862 \\
$\begin{array}{l}\text { LDL-cholesterol } \\
\quad(\mathrm{mg} / \mathrm{dL})\end{array}$ & & & \\
$\begin{array}{l}\text { HDL-cholesterol } \\
\quad(\mathrm{mg} / \mathrm{dL})\end{array}$ & $57.8 \pm 1.4$ & $43.1 \pm 3.7$ & 0.005 \\
Triglyceride (mg/dL) & $162.1 \pm 14.8$ & $210.0 \pm 36.7$ & 0.257 \\
Non-HDL-cholesterol & $119.1 \pm 3.4$ & $109.1 \pm 10.8$ & 0.878 \\
$\quad(\mathrm{mg} / \mathrm{dL})$ & & & \\
HbA1c $(\%)$ & $5.7 \pm 0.1$ & $6.1 \pm 0.4$ & 0.447 \\
EPA (mg/L) & $70.9 \pm 3.7$ & $46.4 \pm 9.9$ & $\mathrm{NA}$ \\
AA (mg/L) & $191.4 \pm 5.2$ & $203.1 \pm 13.9$ & $\mathrm{NA}$ \\
DHA (mg/L) & $164.0 \pm 6.8$ & $154.2 \pm 19.6$ & $\mathrm{NA}$ \\
EPA/AA ratio & $0.392 \pm 0.023$ & $0.236 \pm 0.052$ & $\mathrm{NA}$ \\
$\log$ EPA & $1.779 \pm 0.023$ & $1.590 \pm 0.072$ & 0.017 \\
$\log$ AA & $2.261 \pm 0.012$ & $2.297 \pm 0.030$ & 0.394 \\
$\log$ DHA & $2.178 \pm 0.015$ & $2.159 \pm 0.045$ & 0.727 \\
$\log$ EPA/log AA ratio & $0.788 \pm 0.010$ & $0.693 \pm 0.031$ & 0.007
\end{tabular}

LDL: low-density lipoprotein, HDL: high-density lipoprotein, $\mathrm{Hb}$ : hemoglobin, EPA: eicosapentaenoic acid, AA: arachidonic acid, DHA: docosahechisaenoic acid, NA: not assessed.

patients with coronary artery diseases (6). The contemporary data on fatty acid composition of the general population are not sufficient for discussion. In the Kumihama study, the EPA/AA ratio was reported as about 0.6, where the blood sampling was carried out from 1994 to 1996 (7), indicating that the data are not up to date. Recently we reported that the EPA/AA ratio of the general population was 0.33 , for blood sampling which was performed in 2011 (8). However, it seems to be difficult to directly compare this data with that of the present study because the participants of the general population study were younger than those of the present study. Considering background differences among previous studies, the EPA/AA ratio of the present cohort does not seem to be at an extremely abnormal level.

Comparing with the non-PTE group, the PTE group had slightly but not significantly higher levels in body mass index, triglyceride and $\mathrm{HbA1c}$, and a significantly lower value in HDL-cholesterol. These data indicate that the PTE group, as a whole, might have more features of metabolic syndrome than the non-PTE group. These differences might result in the lower EPA/AA ratio in the PTE group.

So far, some reports have demonstrated that n-3 PUFAs inhibit platelet aggregation (9-11) and reduce the generation of chemical mediators which activate platelet functions $(9,12)$. Notably, a recent study revealed that anti-platelet therapy prevented the recurrence of venous thrombosis (13). Thus, we would like to propose that a low EPA/AA ratio might be one of the underlying conditions to raise platelet activities and that the anti-platelet effect of EPA could have preventive actions against venous thrombosis and PTE. A recent 
Table 4. Multiple comparisons of EPA and EPA/AA ratio.

\begin{tabular}{lccc}
\hline & $\begin{array}{c}\text { non-PTE } \\
(n=132)\end{array}$ & $\begin{array}{c}\text { PTE without carcinoma } \\
(n=6)\end{array}$ & $\begin{array}{c}\text { PTE with carcinoma } \\
(n=6)\end{array}$ \\
\hline EPA $(\mathrm{mg} / \mathrm{L})$ & $70.9 \pm 3.7$ & $33.6 \pm 5.8$ & $59.2 \pm 18.2$ \\
EPA/AA ratio & $0.392 \pm 0.023$ & $0.151 \pm 0.020$ & $0.320 \pm 0.092$ \\
$\log$ EPA & $1.779 \pm 0.023$ & $1.493 \pm 0.076^{*}$ & $1.688 \pm 0.116^{\mathrm{NS}}$ \\
$\log \mathrm{EPA} / \log$ AA ratio & $0.788 \pm 0.010$ & $0.639 \pm 0.027^{*}$ & $0.747 \pm 0.049^{\mathrm{NS}}$
\end{tabular}

EPA: eicosapentaenoic acid, AA: arachidonic acid, *indicates $p<0.01$ compared with the non-PTE group by Bonferroni correction, ${ }^{\mathrm{NS}}$ indicates no significant differences when compared with the non-PTE or the PTE without carcinoma groups.

report, which showed that administration of EPA prevented venous thromboembolism in a rat model (14), further supports our proposal.

Since the presence of carcinoma is one of the risk factors of PTE, we divided the PTE group into two groups by the presence $(n=6)$ or absence $(n=6)$ of malignant carcinoma. Values of EPA and the EPA/AA ratio of the PTE without carcinoma group were $33.6 \mathrm{mg} / \mathrm{L}$ and 0.151 (Table 4). Multiple comparisons for log EPA and $\log$ EPA/log AA ratio among groups of non-PTE, PTE without carcinoma and PTE with carcinoma revealed that the PTE without carcinoma group had a significantly lower log EPA level $(p=0.009)$ and log EPA/ $\log$ AA ratio $(p=0.002)$ than the non-PTE group (Table 4). On the other hand, the PTE with carcinoma group did not show differences in log EPA or log EPA/log AA ratio as compared with the non-PTE group ( $p=0.40$ for $\log$ EPA and $p=0.39$ for $\log$ EPA $/ \log$ AA ratio) (Table 4 ).

In clinical practice, patients with carcinoma are not uniform. They can show a wide spectrum of nutritional status from obesity to cachexia, depending upon the disease stage and the origin of the carcinoma. It is wellrecognized that the intake of $n-3$ PUFAs determines the level of EPA (15). Although the fatty acid composition in malignancy has not been fully elucidated, it is expected to vary widely. Indeed, it seems that some patients with carcinoma had low EPA/AA ratios, and others did not. This phenomenon might have resulted in the failure to show a significant difference in EPA/AA ratio between the groups of PTE with and without carcinoma.

Under these conditions, it is not uncommon that deep vein thrombosis/PTE are associated with cancer. This is known as Trousseau syndrome (16). Secreted factors produced by carcinoma are considered to initiate blood coagulation in this disorder (17). We speculate that the coagulatory disorders caused by carcinoma might affect the formation of venous thrombus, irrespective of the $\mathrm{EPA} / \mathrm{AA}$ ratio. As a result, the EPA/AA ratio in the PTE with carcinoma group might remain statistically comparable to that of the non-PTE group.

There are some limitations to this study. Our study is a single center cross-sectional study and includes a small sample size, indicating the difficulties in deriving definitive evidence. Further clinically interventional studies or biological research is necessary to confirm the findings.

In conclusion, we found that patients with PTE have low serum EPA/AA ratios, although the presence of malignant carcinoma should be taken into account.

\section{REFERENCES}

1) Mozaffarian D, Rimm EB. 2006. Fish intake, contaminants, and human health: evaluating the risks and the benefits. JAMA 296: 1885-1899.

2) GISSI-Prevenzione Investigators. 1999. Dietary supplementation with n-3 polyunsaturated fatty acids and vitamin $\mathrm{E}$ after myocardial infarction: results of the GISSI-Prevenzione trial. Lancet 354: 447-455.

3) Albert MC, Campos H, Stampfer JM, Ridker MP, Manson EJ, Willett CW, Ma J. 2002. Blood levels of long-chain n-3 fatty acids and the risk of sudden death. $N$ Engl J Med 346: 1113-1118.

4) Sekikawa A, Curb JD, Ueshima H, El-Saed A, Kadowaki T, Abbott RD, Evans RW, Rodriguez BL, Okamura T, Sutton-Tyrrell K, Nakamura Y, Masaki K, Edmundowicz D, Kashiwagi A, Willcox BJ, Takamiya T, Mitsunami K, Seto TB, Murata K, White RL, Kuller LH; ERA JUMP (Electron-Beam Tomography, Risk Factor Assessment among Japanese and U.S. Men in the Post-World War II Birth Cohort) Study Group. 2008. Marine-derived n-3 fatty acids and atherosclerosis in Japanese, JapaneseAmerican, and white men: a cross-sectional study. J Am Coll Cardiol 52: 417-424.

5) Lands WEM, Hamazaki T, Yamazaki K, Okuyama H, Sakai K, Goto Y, Hubbard VS. 1990. Changing dietary patterns. Am J Clin Nutr 51: 991-993.

6) Domei T, Yokoi H, Kuramitsu S, Soga Y, Arita T, Ando K, Shirai S, Kondo K, Sakai K, Goya M, Iwabuchi M, Ueeda M, Nobuyoshi M. 2012. Ratio of serum n-3 to n-6 polyunsaturated fatty acids and the incidence of major adverse cardiac events in patients undergoing percutaneous coronary intervention Circ J 76: 423-429.

7) Nakamura T, Azuma A, Kuribayashi T, Sugihara H, Okuda S, Nakagawa M. 2004. Serum fatty acid levels, dietary style and coronary heart disease in three neighbouring areas in Japan: the Kumihama study. Br J Nutr 89: $267-272$.

8) Oshima Y, Niki K, Hiramoto Y, Morimoto S, Takami H, Saito H, Koga M, Izumi M. 2013. Analyses on the serum fatty acid composition of participants in comprehensive medical examination. Med J KINKI Cent Hosp 33: 19-24 (in Japanese).

9) von Schacky C, Fischer S, Weber PC. 1985. Longterm effects of dietary marine omega-3 fatty acids upon plasma and cellular lipids, platelet function, and eicosanoid formation in humans. J Clin Invest $\mathbf{7 6}$ : 1626-1631.

10) Dyerberg J, Bang HO, Stoffersen E, Moncada S, Vane JR. 1978. Eicosapentaenoic acid and prevention of throm- 
bosis and atherosclerosis? Lancet 2: 117-119.

11) Hirai A, Terano T, Hamazaki T, Sajiki J, Kondo S, Ozawa A, Fujita T, Miyamoto T, Tamura Y, Kumagai, A. 1982. The effects of the oral administration of fish oil concentrate on the release and the metabolism of [14C]arachidonic acid and [14C]eicosapentaenoic acid by human platelets. Thromb Res 28: 285-298.

12) Lee TH, Hoover RL, Williams JD, Sperling RI, Ravalese J 3rd, Spur BW, Robinson DR, Corey EJ, Lewis RA, Austen KF. 1985. Effect of dietary enrichment with eicosapentaenoic and docosahexaenoic acids on in vitro neutrophil and monocyte leukotriene generation and neutrophil function. N Engl J Med 312: 1217-1224.

13) Becattini C, Agnelli G, Schenone A, Eichinger S, Bucherini E, Silingard M, Bianchi M, Moia M, Ageno W,
Vandelli MR, Grandone E, Prandoni, R. 2012. Aspirin for preventing the recurrence of venous thromboembolism. N Engl J Med 366: 1959-1967.

14) Kuma H, Nagashima R, Hatae H, Tsuda T, Hamasaki N. 2013. Beneficial effect of EPA (20:5 n-3 PUFA) on preventing venous thromboembolism: A rat tail thrombosis model experiment. Thromb Res 131: 107-108.

15) Ezaki O, Miyake Y, Sato S, Iso H. 2013. Dietary reference intakes for japanese 2010: Fat. J Nutr Sci Vitaminol 59: S44-S52.

16) Trousseau A. 1865. Phlegmasia alba dolens. Clinique Medicale de l'Hotel-Dieu de Paris 3: 654-712.

17) Varki A. 2007. Trousseau's syndrome: multiple definitions and multiple mechanisms. Blood 110: 1723-1729. 